H. Lapp, P. Lauten

Notfallrevaskularisation bei STEMI und kardiogenem Schock

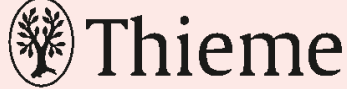




\title{
Notfallrevaskularisation bei STEMI und kardiogenem Schock
}

\author{
Harald Lapp, Philipp Lauten
}

\section{Übersicht}

Einleitung

Diagnose

Allgemeine therapeutische

Maßnahmen

Interventionelle Reperfusion

(primäre $\mathrm{PCl}$ )

\section{Einleitung}

Weltweit ist die koronare Herzkrankheit eine der häufigsten Diagnosen in Bezug auf Morbidität und Mortalität. Der akute Myokardinfarkt mit ST-Streckenhebungen im EKG (STEMI) weist eine Inzidenz von ca. 70/100000/Jahr auf, wobei diese bei einem parallelen Anstieg des Non-STEMI in den letzten Jahren etwas rückläufig war [1]. Wesentliche Prädiktoren für die Mortalität des STEMI sind das Alter, der klinische Zustand bei der Akutaufnahme (Killip-Klasse), die Zeitverzögerung bis zur Therapie, die Art der Therapie, die Komorbidität (früherer Myokardinfarkt, Diabetes, Niereninsuffizienz), das Ausmaß der KHK und die Ejektionsfraktion.

Die primäre perkutane Koronarintervention (PCI) ohne vorherige Fibrinolyse ist die Therapie der Wahl des akuten Myokardinfarkts mit ST-Strecken-Hebungen (STEMI) und für Patienten im infarktbedingten kardiogenen Schock. Mit der PCI kann man das Infarktgefäß schneller wiedereröffnen und einen uneingeschränkten Koronarfluss (TIMI 3) zuverlässiger wiederherstellen. Im Vergleich zur konservativen (Fibrinolyse-)Therapie ist die Rate an erneuten Verschlüssen des Infarktgefäßes zudem geringer. Die konsequente Umsetzung der invasiven Strategie resultiert daher auch in einer geringeren Morbidität und Mortalität. Voraussetzungen hierfür ist jedoch die konsequente Umsetzung bestimmter Kriterien:
- rasche Diagnostik

- Einhaltung von Zeitlimits

- rasche interventionelle Reperfusion mit optimalem Primärergebnis (TIMI-3-Fluss)

- optimale peri- und postinterventionelle Begleittherapie

Die Überlegenheit der invasiven Strategie im Vergleich zum konservativen Vorgehen ist in zahlreichen kontrollierten Studien belegt und hat als evidenzbasierte Medizin Eingang in die Leitlinien der unterschiedlichen Fachgesellschaften gefunden. Die Leitlinien der European Society of Cardiology (ESC) und der American Heart Association/des American College of Cardiology (AHA/ACC) bilden die Grundlage dieses Updates. Sie werden vorausgesetzt und sollen hier nicht wiederholt werden. Dieses Update soll Aspekte diskutieren, die einerseits noch immer Schwierigkeiten bei der Umsetzung der Leitlinien bereiten oder für die es zum Zeitpunkt der Leitlinienerstellung noch eine sehr unklare Datenlage gab.

\section{Diagnose}

\section{Kriterien}

Eine rasche Diagnostik ist ein kritischer Aspekt in der erfolgreichen Behandlung des STEMI, zumal sich ca. ein Drittel der Patienten mit atypischen Beschwerden und 
Befunden präsentiert. Einige Aspekte der Diagnostik sollen zusammengefasst wiederholt werden.

Die Diagnose eines akuten Myokardinfarkts beruht auf folgenden Kriterien:

- der signifikante Anstieg oder Abfall von Biomarkern, bevorzugt hochsensitives Troponin in Verbindung mit

- Symptomen einer Myokardischämie

- neuen oder wahrscheinlich neuen ST-Streckenveränderungen im EKG oder ein neuer Linksschenkelblock

- im Verlauf die Entwicklung von Q-Zacken im EKG

- der Nachweis einer Myokardnekrose/-funktionseinschränkung in der Bildgebung

- Identifizierung eines akuten Koronarverschlusses

(Thrombus/Plaqueruptur) im Herzkatheter

\section{EKG-Diagnostik}

Für die Diagnose des STEMI ist der Nachweis von ST-

Streckenveränderungen im 12-Kanal-EKG wesentlich. Für ein positives 12-Kanal-EKG gelten folgende Kriterien:

- neue ST-Strecken-Hebungen $\geq 0,1 \mathrm{mV}$ am J-Punkt in allen Ableitungen

- (außer in $V_{2}$ bis $V_{3}$, hier gelten $\geq 0,2 \mathrm{mV}$ bei Männern $>40$ Jahre, $\geq 0,25 \mathrm{mV}$ bei Männern $<40$ Jahre und $\geq 0,15 \mathrm{mV}$ bei Frauen)

STEMI-Äquivalente sind:

- ein neu aufgetretener Linksschenkelblock

- neue ST-Hebungen $\geq 0,1 \mathrm{mV}$ in den Ableitungen $V_{7}$ bis $\mathrm{V}_{9}$ (inferobasaler Infarkt)

- neue ST-Hebungen $\geq 0,1 \mathrm{mV}$ in den Ableitungen $V_{3} R$ und $\mathrm{V}_{4} \mathrm{R}$ (Rechtsherzinfarkt)

- neue ST-Hebungen $\geq 0,05 \mathrm{mV}$ in aVR mit gleichzeitigen ST-Senkungen $\geq 0,05 \mathrm{mV}$ in mehreren benachbarten Ableitungen

Problematisch ist die STEMI-Diagnose bei Linksschenkelblock. Grundsätzlich unterscheidet sich der neu aufgetretene nicht vom vorbestehenden Linksschenkelblock. Aber auch bei Linksschenkelblock erlauben die sehr spezifischen, leider wenig sensitiven Sgarbossa-Kriterien die Diagnose eines STEMI [4, 5]. Den Kriterien liegt die Analyse der EKGs der GUSTO-I-Studie zugrunde. Drei unabhängige EKG-Kriterien wurden definiert:

- ST-Hebungen $\geq 1 \mathrm{~mm}$, konkordant mit dem QRSKomplex in einer Ableitung (5 Punkte)

- ST-Senkungen $\geq 1 \mathrm{~mm}$ in den Ableitungen $\mathrm{V}_{1}, \mathrm{~V}_{2}$ oder $\mathrm{V}_{3}$ (3 Punkte)
- ST-Hebungen $\geq 5 \mathrm{~mm}$ diskordant mit QRS-Komplex in einer Ableitung (2 Punkte)

Ein Score von $\geq 3$ Punkten hat eine Spezifität von 95\% bei allerdings geringer Sensitivität.

Bei Patienten, bei denen die Diagnose nicht eindeutig gestellt werden kann, sind serielle EKGs, hs-Troponin-Bestimmungen und eine BedsideEchokardiografie für die Bestätigung bzw. den Ausschluss der Diagnose erforderlich.

Bei klarer Anamnese und eindeutigem EKG sollte allerdings keine Zeit bis zur Reperfusion durch weitere Diagnostik (z. B. Biomarker) verschenkt werden. Hochsensitives Troponin (T oder I) ist der Biomarker der Wahl mit einer hohen Sensitivität und Spezifität für eine Myokardnekrose.

\section{Allgemeine therapeutische Maßnahmen}

\section{Prähospitalphase}

Die Behandlung des STEMI beginnt bereits in der prähospitalen Phase. Die symptomatische Behandlung von Schmerz, Brechreiz, Angst und Luftnot sind wichtige ergänzende Therapiemaßnahmen:

- Das am häufigsten eingesetzte Analgetikum ist (fraktioniert gegebenes) Morphin.

- Sauerstoff sollte bei Patienten mit Dyspnoe oder einer Hypoxie $\left(\mathrm{SaO}_{2}<95 \%\right)$ eingesetzt werden. Eine „Luxusoxygenierung“ ist dabei nicht sinnvoll, zumal es Hinweise auf negative Effekte in der Reperfusionsphase gibt.

Ein kontiniuerliches EKG-/Rhythmus-Monitoring ist obligat. Bereits prähospital müssen Überlegungen bzw. Vorbereitungen zu einer frühzeitigen Reperfusionstherapie angestellt werden. Die Gesamtbehandlung muss so organisiert sein, dass vom ersten medizinischen Kontakt bis zur Drahtpassage durch den Koronarverschluss nicht mehr als 90 Minuten vergehen. Bei Patienten, die sich früh vorstellen und Zeichen eines „großen“ Myokardinfarkts bieten, sollten dies sogar nur 60 Minuten sein [6]. Eine Klinik mit PCI-Möglichkeit muss so organisiert sein, dass zwischen Eintreffen des Patienten und PCI keine 60 Minuten vergehen („doorto-balloon-Zeit“"). Diese Zeiten gelten als Mindestanforderungen. Jede Klinik mit PCI-Möglichkeit sollte sich gemeinsam mit dem regionalen Rettungsdienst so 
organisieren, dass diese Zeiten so kurz als irgend möglich sind. Patienten, die eindeutige Kandidaten für eine primäre PCI sind, sollten unmittelbar vom Rettungsdienst in ein Katheterlabor verbracht werden, um keine Zeit zu verlieren. Bei Patienten, deren Infarktbeginn mehr als 12 Stunden zurückliegt, ist die Datenlage nicht eindeutig: In kleineren Studien hat die erfolgreiche PCI bei Patienten, die sich ohne anhaltende Ischämie zwischen 12 und 48 Stunden vorstellen, vitales Myokard erhalten und zu einem besseren Überleben beigetragen [7]. In der großen OAT-Studie (OAT = „occluded artery trial“) zeigte sich dagegen keinen Vorteil gegenüber der alleinigen medikamentösen Therapie [8].

\section{Indikationen}

Die Indikationen für eine sofortige Reperfusionstherapie bei STEMI sind:

- sofortige Reperfusion bei Symptomen < 12 Stunden und ST-Hebungen im EKG bzw. einem ST-Hebungsäquivalent

- sofortige Reperfusion bei ST-Hebungen>12 Stunden und anhaltenden Symptomen bzw. anderen Zeichen der anhaltenden Ischämie

- in Erwägung zu ziehende Reperfusion bei asymptomatischen Patienten mit Symptomen zwischen 12 und 24 Stunden

- individuelle Entscheidung bei Patienten mit Symptomen $>24$ Stunden

\section{Interventionelle Reperfusion (primäre $\mathrm{PCl}$ )}

Die primäre $\mathrm{PCI}$ ist die bevorzugte Reperfusionsstrategie bei STEMI. Sie ist definiert als die notfallmäßige PCI im Rahmen des STEMI ohne vorausgehende Fibrinolyse. Zahlreiche Studien haben die Überlegenheit der interventionellen Reperfusion gegenüber der Fibrinolyse belegt $[9,10,11]$.

Die wesentlichen Vorteile der primären PCI sind

- die höhere Erfolgsrate in der Wiedereröffnung des Infarktgefäßes (>90\%)

- die geringe Rate erneuter Verschlüsse

- die geringere Rate an schweren Blutungskomplikationen
Diese Vorteile resultieren in einer geringeren Mortalität, vorausgesetzt, dass kein Zeitverlust in der Reperfusion durch die $\mathrm{PCI}$ verursacht wird.

Im Folgenden sollen einzelne prozedurale Aspekte der primären $\mathrm{PCI}$ vor dem Hintergrund neuer Daten diskutiert werden.

\section{Stents und bioresorbierbare Gefäßstützen}

Wie bei elektiven Interventionen reduzieren medikamentenbeschichtete Stents („drug eluting stent“, DES) auch bei der primären PCI im Vergleich zu unbeschichteten Stents („bare metal stent“, BMS) das Risiko einer signifikanten erneuten Stenose und die Notwendigkeit einer erneuten Intervention im gleichen Gefäß [12] Der ursprüngliche Verdacht, dass DES zu einer erhöhten Rate an frühen Stentthrombosen und damit zu einer erhöhten Mortalität führen könnten, hat sich insbesondere seit dem Einsatz moderner DES der 2. Generation nicht bestätigt. Die Dauer der dualen Plättchenhemmung wird im Fall der PCI beim akuten Koronarsyndrom nicht durch den implantierten Stent, sondern durch das Krankheitsbild bestimmt. Sie sollte über $\geq 12$ Monate gegeben werden.

Der Einsatz bioresorbierbarer Gefäßstützen („bioresorbable vascular scaffold“, BVS) hat einige interessante theoretische Aspekte bei der interventionellen Behandlung des akuten Koronarsyndroms [13]. Die Ergebnisse der TROFI-II-Studie unterstützen das Konzept, dass ein BVS vor dem Hintergrund der spezifischen Pathophysiologie im Koronarverschluss ideal sei, um langfristig eine optimale Gefäßanatomie wiederherzustellen [14]. Studien mit klinischen Endpunkten im Vergleich zu modernen DES fehlen, sodass der generelle Einsatz zum jetzigen Zeitpunkt noch nicht empfohlen werden kann.

Der generelle Einsatz von BVS kann zum jetzigen

Zeitpunkt nicht empfohlen werden.

\section{Radialer vs. femoraler Gefäßzugang}

Periprozedural ist in der Therapie des akuten Koronarsyndroms und insbesondere auch des STEMI eine intensivierte Antikoagulation und Plättchenhemmung erforderlich, die das Risiko für Blutungen erhöht. Die Blutung ist ein wesentlicher Prädiktor für die Morbidität, aber auch für die Mortalität in der Therapie des STEMI [15]. Dies hat konsequenterweise auch Auswir- 
kungen auf den arteriellen Gefäßzugang bei der primären PCI. Zahlreiche Studien zeigen, dass der transradiale Zugang die Zahl relevanter Blutungen reduziert und damit zu einer verminderten Mortalität führen kann $[16,17]$. Die prozedurale Erfolgsrate hängt aber eindeutig von der Erfahrung mit dem radialen Zugang ab: Sie ist nur in trainierten Händen vergleichbar hoch wie beim transfemoralen Zugang [18].

Der transradiale Zugang ist inbesondere bei der Versorgung des STEMI zu bevorzugen. Im Notfall sollte jedoch der Untersucher den für ihn sichersten Zugang wählen (femoral oder transradial).

\section{Aspirationsthrombektomie}

In zahlreichen Studien war eine höhere Thrombuslast im Infarktgefäß bei STEMI mit einem erhöhten Risiko für „no-reflow“, größere transmurale Nekrosen, distale Embolien, Stent-Thrombosen und ggf. Mortalität assoziiert. Daher lag es nahe, vor der primären PCI nach Drahtpassage zunächst eine Aspirationsthrombektomie durchzuführen.

Die Ergebnisse der randomisierten TAPAS-Studie (TAPAS = „thrombus aspiration during primary percutaneous coronary intervention in acute myocardial infarction study“) und anderer kleinerer Studien deuteten darauf hin, dass eine routinemäßige Thrombusaspiration vor der Angioplastie die Ergebnisse der primären PCI verbessern könnten [19,20]. Neuere Studien konnten diesen klaren Vorteil nicht mehr belegen [21, 22,23]. Auch eine aktuelle Metaanalyse mit der Auswertung von über 20000 Patienten ergab für den routinemäßigen Einsatz der Aspirationsthrombektomie keinen Vorteil hinsichtlich Mortalität, erneutem Infarkt oder Stentthrombose [24].
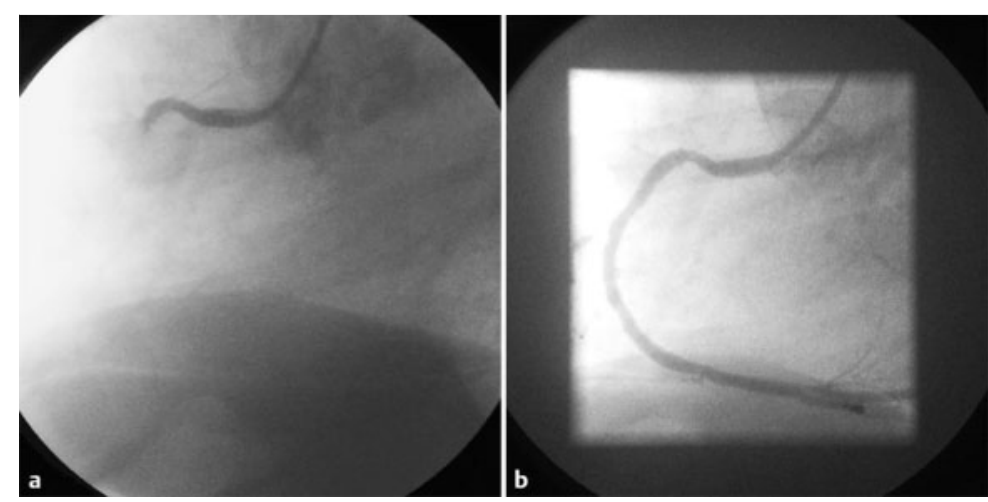

Abb. 1 Thrombotischer In-Stent-Verschluss der RCA (a) und Angiografie nach Thrombektomie (b).
Unabhängig davon bleibt der individualisierte Einsatz der Aspirationsthrombektomie z.B. eine klinische Option bei:

- persistierenden großen Thromben nach Ballonangioplastie und vor Stentimplantation

- distalen Embolien

Im Einzelfall kann man dadurch das primäre klinische und angiografische Resultat verbessern. Eine spezifische Indikation könnte die akute Stent-Thrombose sein (Abb. 1, Abb. 2).

\section{Infarkt-Gefäß-PCI vs. Mehrgefäß-PCI}

Die Hälfte der STEMI-Patienten hat eine Mehrgefäßerkrankung [25]. Grundsätzlich sind dabei unterschiedliche Revaskularisationsstrategien möglich:

- alleinige primäre PCI der infarktauslösenden Läsion („culprit lesion“)

- primäre PCI der „culprit lesion“ sowie aller anderen hochgradigen Stenosen in der gleichen Sitzung

- primäre PCI der „culprit lesion“ und PCI der ischämieauslösenden Läsionen noch im gleichen Aufenthalt nach Ischämiediagnostik vor Entlassung

- primäre PCI der „culprit lesion“ und PCI der ischämieauslösenden Läsionen in einer chronischen Phase nach Entlassung und Ischämiediagnostik

Die verfügbaren Daten zu den unterschiedlichen Strategien sind widersprüchlich, da die verschiedenen Studien unterschiedliche Kollektive eingeschlossen und nach ganz unterschiedlichen Protokollen behandelt haben $[25,26,27,28,29,30]$.

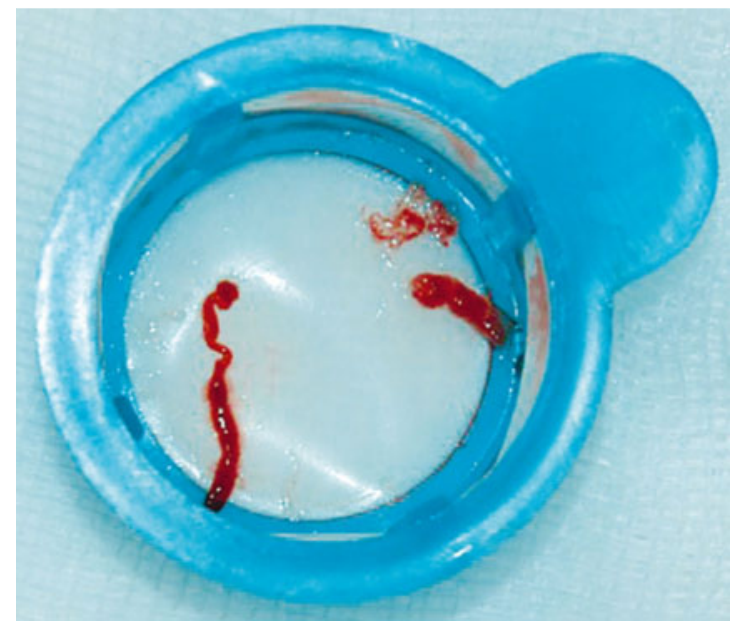

Abb. 2 Aspirationsthrombektomie bei akutem Stentverschluss nach vorzeitigem Beenden einer dualen Plättchenhemmung. 
Die bisherigen Leitlinien unterschiedlicher Fachgesellschaften enthalten keine Empfehlung für die PCI der nicht auslösenden Stenosen bei stabilen Patienten $[2,3]$. Nur bei instabilen Patienten oder in bestimmten Hochrisikosituationen wird die PCI der nicht infarktverursachenden Läsionen erwogen. Die wesentliche Rationale für diese Empfehlungen waren:

- das erhöhte Risiko periprozeduraler Komplikationen,

- höhere Kontrastmittelmengen mit dem Risiko des akuten Nierenversagens,

- längere Prozedurdauern und

- Probleme einer optimalen Steuerung der Antikoagulation.

Die Ergebnisse aktueller randomisierter Studien deuten darauf hin, dass ausgewählte Patienten klinisch von einer primären Mehrgefäß-PCI oder einer Zweiteingriff-PCI vor Entlassung profitieren können [28,29,31]. Untersucht wurden dabei kombinierte Endpunkte aus Mortalität, Rehospitalisation, Angina-Symptomatik und anderen Endpunkten. Die Ergebnisse dieser Studien erlauben keine generelle Empfehlung für eine Mehrgefäß-PCI im Rahmen des STEMI. Sie zeigen aber, dass diese Prozedur unter Berücksichtigung klinischer Variablen sicher durchgeführt werden kann. Die Entscheidung richtet sich vor allem nach der Symptomatik und der Koronarmorphologie, aber auch nach nicht kardialen Befunden wie der Nierenfunktion oder Indikationen zur Antikoagulation. Diese Aussagen beziehen sich auf das Vorgehen bei hämodynamisch stabilen Patienten.

Das Vorgehen im kardiogenen Schock ist ebenfalls offen. In der derzeit laufenden CULPRIT-SHOCK-Studie wird die Mehrgefäß-PCI im kardiogenen Schock mit einem abgestuften Vorgehen randomisiert verglichen [32]. Die primären Endpunkte der Studie sind die 30Tage-Mortalität sowie das akuten Nierenversagen mit der Notwendigkeit einer Nierenersatztherapie. Ein Follow-up nach 6 und 12 Monaten ist geplant. Die Studie wird voraussichtlich Anfang 2017 abgeschlossen sein.

Ausgewählte Patienten können von einer primären Mehrgefäß-PCl oder einer Zweiteingriff-PCl vor Entlassung profitieren. Welche Patienten dies sind, richtet sich vor allem nach der Symptomatik und der Koronarmorphologie, aber auch nach nicht kardialen Befunden wie der Nierenfunktion oder Indikationen zur Antikoagulation.

\section{Duale Plättchenhemmung}

Die duale Plättchenhemmung (DAPT) ist eine Kombination aus ASS $100 \mathrm{mg}$ und einem $\mathrm{P}_{2} \mathrm{Y}_{12}$-Rezeptorhemmer (Clopidogrel, Prasugrel, Ticagrelor). Sie ist fester Bestandteil der Therapie eines akuten STEMI, insbesondere nach interventioneller Therapie.

Es gibt eine gute Evidenz, dass bei Patienten mit STEMI, die mit einer PCI und Stent versorgt werden, Ticagrelor bzw. Prasugrel im Vergleich zu Clopidogrel klinische Vorteile bieten [33,34]. Prasugrel ist bei Patienten nach Schlaganfall oder TIA in der Anamnese kontraindiziert und soll bei Patienten mit einem Körpergewicht unter $60 \mathrm{~kg}$ bzw. einem Alter von 75 Jahren oder mehr nicht gegeben werden. Ticagrelor und Prasugrel sind bei Patienten nach intrazerebraler Blutung kontraindiziert. Die DAPT nach STEMI kann mit Clopidogrel durchgeführt werden, wenn Kontraindikationen gegen neuere ADP-Antagonisten bestehen oder besondere patientenindividuelle Umstände vorliegen.

Die orale Startdosis beträgt für:

- Prasugrel $60 \mathrm{mg}$ (Fortsetzung mit $1 \times 10 \mathrm{mg} / \mathrm{d}$ oder $1 \times 5 \mathrm{mg} / \mathrm{d}$ bei besonderen Risikokonstellationen)

- Ticagrelor $180 \mathrm{mg}$ (Fortsetzung mit $2 \times 90 \mathrm{mg} / \mathrm{d}$ )

- Clopidogrel 600 mg (Fortsetzung mit $1 \times 75$ mg/d)

Bei Clopidogrel ist die frühzeitige, bereits prähospitale Gabe der Startdosis vorteilhaft. Dagegen werden die neueren, sehr schnell wirksamen oralen Plättchenhemmer erst intrahospital bzw. periprozedural nach Kenntnis des Koronarbefundes gegeben. Für Ticagrelor und Prasugrel liegen zur Frage der prähospitalen bzw. präprozeduralen Gabe widersprüchliche Befunde vor $[35,36]$ : Die prähospitale Gabe scheint sicher zu sein und führt auch in pharmakologischen Untersuchungen zu einer früheren Hemmung der Plättchenaktivität. Ein klinischer Gewinn für die Patienten konnte bisher aber nicht nachgewiesen werden.

Cangrelor ist der erste intravenöse und schnell reversible $\mathrm{P}_{2} \mathrm{Y}_{12}$-Rezeptorhemmer mit einer kurzen Halbwertszeit unter 5 Minuten. In einer der 3 randomisierten Studien konnte gegenüber der alleinigen Therapie mit Clopidogrel eine Reduktion periprozeduraler Myokardinfarkte und Stentthrombosen gezeigt werden [37]. Theoretisch erscheint eine parenterale Vorbehandlung mit Cangrelor sinnvoll, der klinische Nutzen insbesondere im Vergleich zu Prasugrel und Ticagrelor ist noch nicht gezeigt. 
Alle Patienten unter DAPT mit gastrointestinalen Blutungen in der Anamnese oder mit einer zusätzlichen oralen Antikoagulation (Triple-Therapie), Steroiden oder nicht steroidalen Antirheumatika (NSAR) sollten Protonenpumpenhemmer erhalten.

Patienten mit einem akuten Koronarsyndrom haben auch längerfristig ein erhöhtes Risiko für kardiovaskuläre Ereignisse, sodass eine verlängerte Gabe von P2Y12-Rezeptorhemmern untersucht wurde:

- In der PEGASUS-TIMI-54-Studie wurde die ASSMonotherapie gegen eine verlängerte DAPT mit Ticagrelor (60 bzw. $90 \mathrm{mg}$ zweimal täglich) verglichen [38]: Einerseits waren kardiovaskuläre Ereignisse signifikant reduziert, andererseits die Rate an Blutungen erhöht - allerdings ohne signifikanten Anstieg fataler oder intrakranieller Blutungen.

- Andere Studien und eine Metaanalyse konnten diese Ergebnisse wiederholen [39,40,41], wobei insbesondere kardiovaskuläre Hochrisikopatienten von einer verlängerten DAPT profitieren.

Zusammenfassend sollte eine DAPT daher für mindestens 6 Monate, in der Regel 12 Monate nach einem STEMI durchgeführt werden. Für ausgewählte Patienten mit einem hohen Ischämie- und einem niedrigen Blutungsrisiko kann eine verlängerte DAPT in einer individuellen Nutzen-RisikoBewertung erwogen werden (Tab. 1).

In einer aktuellen Arbeit wird ein Score für die NutzenRisiko-Bewertung einer verlängerten DAPT vorgeschlagen (DAPT-Score, Tab. 2) [42].

\section{Tabelle 1}

\section{Klinische Parameter für die Nutzen-Risiko-Abwägung einer verlänger-} ten DAPT.

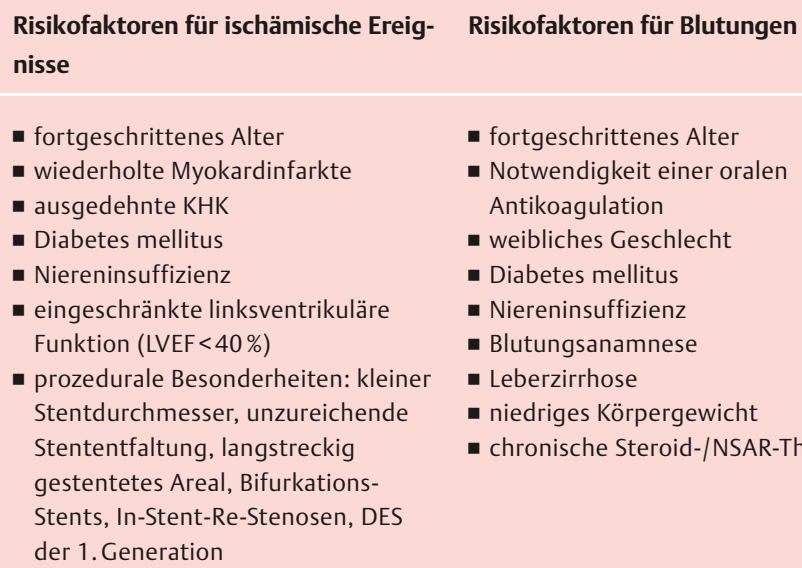

\section{Tabelle 2}

\section{DAPT-Score.}

\begin{tabular}{|l|l|}
\hline Variable & Punkte \\
\hline Alter $\geq 75$ Jahre & -2 \\
\hline Alter 65 bis $<75$ Jahre & -1 \\
\hline Alter $<65$ Jahre & 0 \\
\hline aktiver Raucher & 1 \\
\hline Diabetes mellitus & 1 \\
\hline Myokardinfarkt bei Aufnahme & 1 \\
\hline frühere PCI oder Myokardinfarkt & 1 \\
\hline Stentdurchmesser<3 mm & 1 \\
\hline Paclitaxel-DES & 1 \\
\hline Herzinsuffizienz (CHF) oder LVEF $<30 \%$ & 2 \\
\hline Venenbypass-PCI & 2 \\
\hline
\end{tabular}

Score $<2$ Punkte mit schlechtem Nutzen-/Risiko-Verhältnis $\mathrm{PCI}=$ Perkutane Koronarintervention, $\mathrm{LVEF}=$ linksventrikuläre Ejektionsfraktion

\section{Antikoagulation}

Neben der frühzeitigen dualen Plättchenhemmung (ASS plus $\mathrm{P}_{2} \mathrm{Y}_{12}$-Hemmer) ist insbesondere die frühzeitige parenterale Antikoagulation noch vor Beginn der invasiven Prozedur wichtig. Unfraktioniertes Heparin, Enoxaparin und Bivalirudin sind die therapeutischen Optionen:

- Wenn auch für unfraktioniertes Heparin keine Daten aus kontrollierten Studien vorliegen, gibt es doch eine sehr lange Tradition und Erfahrung vor dem Hintergrund der pathophysiologischen Rationale. Die Dosis an unfraktioniertem Heparin entspricht mit 70-100 IE/kgKG der Dosis bei einer elektiven PCI.

- In einer randomisierten Studie ergab sich für Enoxaparin (initialer i.v. Bolus, gefolgt von einer s.c. Gabe) im Vergleich zu unfraktioniertem Heparin eine niedrigere Rate an Blutungskomplikationen. Die Effektivität in einem kombinierten Endpunkt blieb ohne Unterschiede [43].

- In einer großen randomisierten Studie, HORIZONAMI, war die periinterventionelle Antikoagulation mit Bivalirudin der routinemäßigen Kombination aus unfraktioniertem Heparin und GP-IIb/IIIa-Rezeptorantagonisten hinsichtlich Blutungsereignissen und 
Gesamtmortalität überlegen, obwohl es in der Frühphase mehr koronare Frühverschlüsse gab [44].

Zahlreiche Untersuchungen haben in unterschiedlichen Szenarien Vorteile für die regelmäßige Gabe der GP-Ilb/Illa-Antagonisten Abciximab, Tirofiban oder Eptifibatide im Vergleich zur alleinigen Antikoagulation mit Heparin gesehen. Die aktuellen europäischen und amerikanischen Leitlinien bewerten den Einsatz von GP-IIb/IIIa-Rezeptorantagonisten als Bail-out-Therapie, falls sich angiografisch massive Thromben darstellen oder wenn der koronare Fluss verzögert ist, obwohl der Verschluss beseitigt wurde.

Die Interpretation der Daten zur periinterventionellen Antikoagulation ist schwierig, da keine Studien in Kombination mit den derzeit eingesetzten sehr viel effektiveren oralen Plättchenhemmern vorliegen. Offen ist auch die Frage, ob ergänzend zur Plättchenhemmung eine verlängerte postprozedurale Antikoagulation erforderlich ist. Die Bivalirudin-Studien wurden aktuell zu dieser Frage ausgewertet und es fand sich kein Vorteil für eine verlängerte effektive Antikoagulation vor dem Hintergrund einer erhöhten Blutungsrate [45]. Ob diese Daten auf andere Antikoagulationsregimes insbesondere auch vor dem Hintergrund der neuen Plättchenhemmer übertragbar sind, ist offen. Für die Zeiten der Bettruhe ist zumindest eine Thromboseprophylaxe angezeigt.

\section{Patienten nach kardiopulmonaler Reanimation}

In den ersten Stunden eines STEMI ist der plötzliche Herztod mit Kammerflimmern eine häufige Komplikation insbesondere im präklinischen Umfeld. Bei Patienten, die nach erfolgreicher Reanimation im EKG STHebungen oder Äquivalente zeigen, ist eine unmittelbare Koronarangiografie mit interventioneller Reperfusion indiziert [46]. Liegen dagegen keine eindeutigen EKG-Veränderungen vor, sollte unmittelbar eine Echokardiografie durchgeführt werden. Wandbewegungsstörungen sind zwar nicht spezifisch für eine akute Ischämie, sondern können auch Folge alter Ischämien oder eines Schenkelblocks sein - trotzdem hilft die Echokardiografie dabei, andere Differenzialdiagnosen wie z.B. eine Lungenembolie, eine Perikardtamponade oder eine Aortendissektion auszuschließen. Die notfallmäßige CT-Angiografie bietet eine sichere Differenzierung zur Lungenembolie und Aortendissektion. In unserem Haus werden Postreanimationspatienten von einem spezialisierten Team in der Notaufnahme in

Empfang genommen. Das Team führt eine Echokardiografie durch und entscheidet interdisziplinär, ob noch eine CT-Untersuchung erforderlich ist oder der Patient unmittelbar in ein Katheterlabor verlegt wird. Bei Patienten nach prähospitaler Reanimation infolge Kammerflimmerns wird ergänzend zur Reperfusion eine therapeutische Hypothermie zur Reduktion der neurologischen Folgeschäden durchgeführt.

Patienten nach prähospitaler Reanimation sollten interdisziplinär im ROSC-Team (Intensivmediziner) Kardiologen)behandelt werden. Die milde therapeutische Hypothermie stellt einen Grundpfeiler der Behandlung der Post-Reanimationserkrankung dar.

\section{STEMI-bedingter kardiogener Schock}

Ein kardiogener Schock entsteht bei ca. 6-10\%

aller Patienten mit STEMI und hat mit bis zu $50 \%$ eine hohe Mortalität.

\section{Info für die Praxis}

Interventionelle Aspekte beim stabilen Patienten

- Die primäre PCI ist der Fibrinolyse überlegen.

- Die Implantation eines DES wird bevorzugt.

- Der radiale Zugangsweg sollte (im Vergleich zum femoralen Zugang) bevorzugt werden.

- Die routinemäßige Thrombusaspiration bringt keinen Vorteil.

- Die Beschränkung auf die „culprit lesion“ ist für den Großteil der Patienten sinnvoll.

Periinterventionelle Pharmakotherapie beim stabilen Patienten

- Die Leitlinien bevorzugen Bivalirudin (im Vergleich zu unfraktioniertem Heparin \pm GP-IIb/IIlaAntagonisten).

- Enoxaparin (i. v. Bolus und nachfolgend s.c. Gabe) scheint der alleinigen Gabe von unfraktioniertem Heparin überlegen zu sein.

- Unfraktioniertes Heparin \pm GPIlb/IIla-Antagonisten bekommen alle Patienten, die nicht mit Bivalirudin oder Enoxaparin behandelt werden.

- GP-IIb/IIla-Antagonisten (Abciximab, Tirofiban, Eptifibatide) werden individuell nach Befund gegeben.

- Die potenten und schnell wirksamen $\mathrm{P}_{2} \mathrm{Y}_{12}$-Hemmer (Prasugrel und Ticagrelor) werden gegenüber Clopidogrel bevorzugt.

- Clopidogrel wird nur bei Kontraindikationen für Prasugrel und Ticagrelor gegeben.

- Die routinemäßige prähospitale Vorbehandlung mit den potenten und schnell wirksamen $\mathrm{P}_{2} \mathrm{Y}_{12}$ Hemmern wird nicht empfohlen. 


\section{Revaskularisation}

Die frühzeitige Revaskularisation, interventionell oder chirurgisch, ist die wesentliche Maßnahme zur Senkung der hohen Mortalität. Sie ist der medikamentösen Therapie überlegen, was sowohl in der SHOCK-Studie [48] als auch im Register der SHOCK-Studie gezeigt wurde [49]. Aber auch bei einer erfolgreichen Reperfusion erleiden zahlreiche Patienten einen Schock - insbesondere Patienten über 75 Jahre. Zahlreiche kleinere Studien deuten darauf hin, dass im Fall des kardiogenen Schocks nicht nur die „culprit lesion“ interveniert, sondern eine komplette Revaskularisation angestrebt werden sollte. Dies hat als Empfehlung individuell Eingang in die Leitlinien gefunden [3]. Die Richtigkeit dieser Hypothese und Reperfusionsstrategie wird derzeit in der CULPRIT-Shock-Studie untersucht [32].

\section{Ursachen}

Die wichtigsten Ursachen des kardiogenen Schocks sind beim Vorderwandinfarkt der Verlust an kontraktilem Myokard und beim Hinterwandinfarkt die rechtsventrikuläre Dysfunktion oder akute Störungen des Reizleitungssystems. Andere Schockursachen beim STEMI wie die akute Mitralklappeninsuffizienz bei Papillarmuskeldysfunktion oder -ruptur, der akute Ventrikelseptumdefekt oder die Ruptur der freien Wand treten in der Regel erst 3-5 Tage nach dem STEMI auf. Im Zeitalter der kompletten interventionellen Revaskularisation sind diese Komplikationen deutlich seltener geworden. Das Auftreten mechanischer Infarktkomplikationen erfordert die unmittelbare interdisziplinäre Diskussion im „Heart Team“.

Bei ca. drei Viertel aller Patienten mit kardiogenem Schock ist der akute Myokardinfarkt die Ursache [47].

\section{Diagnose}

Der Schock entwickelt sich meist in den Stunden nach dem Akutereignis. Die wesentlichen klinischen und hämodynamischen Kriterien sind Tachykardie, Hypotonie, niedriges Herzzeitvolumen, erhöhte Füllungsdrücke und Organdysfunktionen (z. B. Oligurie, Anurie, Orthopnoe). Die Notwendigkeit, positiv inotrope Medikamente sowie Vasopressoren zu geben, sind andere Kriterien.
Die wichtigsten Maßnahmen sind:

- die unmittelbare bettseitige Echokardiografie, ggf. transösophageal, zum Erfassen der Ejektionsfraktion oder mechanischer Komplikationen

- die hämodynamische Quantifizierung mit Messung der Füllungsdrücke (LVEDP) im Rahmen der Herzkatheteruntersuchung

- die Messung des Herzzeitvolumens (z.B. invasiv, echokardiografisch)

Auf dieser Datenbasis kann dann individuell entschieden werden, ob neben allgemeinen Maßnahmen eine rein pharmakologische Schocktherapie ausreichend ist oder eine mechanische Unterstützung erforderlich wird.

\section{Allgemeine Maßnahmen beim kardiogenen Schock}

Allgemeine Maßnahmen haben das Ziel, den Stress des Patienten und die Atemarbeit zu reduzieren - und so mittelbar auch den myokardialen Sauerstoffbedarf. Die Gabe von Sauerstoff ist eine wichtige Basismaßnahme bei allen Patienten mit einer $\mathrm{SaO}_{2}<95 \%$. Pharmakologisch stehen Opiate im Vordergrund. Mit Sedativa, insbesondere Benzodiazepinen, sind sie wegen der möglichen Atemdepression nur mit Vorsicht zu kombinieren. Eine frühzeitige Beatmung reduziert die Atemarbeit. Eine nicht invasive Beatmung sollte nur dann umgesetzt werden, wenn der Patient stressfrei beatmet werden kann. Ansonsten ist auch frühzeitig auf eine invasive Beatmung zu wechseln. Die Narkoseeinleitung erfordert viel Erfahrung, um zusätzliche akute Hypotonien zu vermeiden.

\section{Medikamentöse Therapie des kardiogenen Schocks}

Die medikamentöse Therapie besteht aus einer Kombination aus Vasopressor und positiv inotrop wirkenden Substanzen. Der Vasopressor der Wahl ist Noradrenalin, wobei ein arterieller Mitteldruck $>60 \mathrm{mmHg}$ angestrebt wird. Neben der Erhöhung des peripheren Widerstands muss aber auch das Herzzeitvolumen durch positiv inotrope Wirkprinzipien angehoben werden. Die wichtigsten Substanzen dafür sind Dobutamin, Phosphodiesterasehemmer (PDE-3-Hemmer) und Levosimendan. Die 3 Substanzen haben unterschiedliche Wirkprinzipien. PDE-3-Hemmer und Levosimendan führen zusätzlich zu einer Vasodilatation und müssen daher mit einem Vasopressor kombiniert 
werden [50]. Auf initiale Bolusgaben sollte bei beiden Medikamenten bei dann folgender Hypotonie verzichtet werden.

Während bei anderen Schockformen eine positive Flüssigkeitsbilanz zur Stabilisierung angestrebt wird, scheint dies beim kardiogenen Schock prognostisch ungünstig zu sein [51]. Trotzdem ist in Abhängigkeit vom klinischen Verlauf des Schocks auch eine kalkulierte Volumentherapie unverzichtbar.

\section{Mechanische Unterstützung beim kardiogenen Schock}

Die Wirkprinzipien der mechanischen Unterstützung beim kardiogenen Schock sind unterschiedlich und zielen im Wesentlichen auf die Unterstützung/Entlastung der linksventrikulären Funktion. Grundsätzlich problematisch ist die Therapie des refraktären Schocks mit überwiegendem Versagen der rechtsventrikulären Funktion.

Beim rechtsventrikulär bedingten kardiogenen Schock haben die engmaschige hämodynamische Kontrolle und passagere mechanische Unterstützungssysteme entscheidenden Einfluss auf den Verlauf, da pharmakologische Ansätze limitiert sind [52].

Die intraaortale Ballonpumpe (IABP) führt zu einer diastolischen Augmentation des Blutflusses aus der Aorta, senkt die Nachlast für den linken Ventrikel und steigert die Koronarperfusion. Pumpensysteme wie das Impella-System pumpen Blut aktiv aus dem linken Ventrikel in die Aorta, steigern so das Herzzeitvolumen und reduzieren die Nachlast des linken Ventrikels. Systeme wie das Tandem Heart pumpen oxygeniertes Blut über einen transseptalen Zugang aus dem linken Vorhof in die abdominale Aorta. Damit reduzieren sie vor allem die Vorlast des linken Ventrikels. Über die venoarterielle extrakorporale Membranoxygenierung (ECMO) kann aufgrund des Membranoxygenators und der hohen Flussraten, die erzielt werden können, deoxygeniertes Blut mit Sauerstoff gesättigt werden. Die Organe werden über die retrograde Perfusion via Aorta ausreichend oxygeniert. Der frühzeitige Einsatz mechanischer Unterstützungssysteme kann möglicherweise die Prognose verbessern [53]. Die verschiedenen Systeme unterscheiden sich sehr in der Komplexität der Anwendung und benötigen viel Erfahrung des intensivmedizinischen pflegerischen und ärztlichen Personals. Vor dem Beginn mechanischer Unterstüt- zungssysteme muss auch das Therapieziel verantwortlich überlegt werden.

Die intraaortale Ballonpumpe (IABP) war lange Zeit das einzige verfügbare Unterstützungssystem und wurde über 40 Jahre routinemäßig beim kardiogenen Schock eingesetzt. Transfemoral platziert man dabei einen Ballon in die Aorta, der sich EKG-synchronisiert während der Diastole entfaltet und während der Systole entleert. Es ist gut untersucht, dass das Herzzeitvolumen und die Koronarperfusion gesteigert und die linksventrikuläre Nachlast gesenkt werden können. Der klinische Effekt war lange nicht klar. In die randomisierte IABP-SHOCK-II-Studie wurden insgesamt 600 Patienten eingeschlossen. Der primäre Endpunkt 30-Tage-Mortalität konnte durch den Einsatz der IABP nicht signifikant reduziert werden (39,7\% mit IABP vs. 41,3\% ohne IABP) [54]. Aufgrund dieser Daten wird die IABP derzeit nicht mehr routinemäßig im kardiogenen Schock eingesetzt. Als Indikationen verbleiben:

- das Bridging bis zur herzchirurgischen Versorgung mechanischer Infarktkomplikationen,

- die Verlegung im Schock aus einem Krankenhaus ohne Kathetermöglichkeit nach Thrombolyse in ein Krankenhaus mit der Möglichkeit der mechanischen Reperfusion oder

- junge Patienten unter 50 Jahre.

Die mikroaxiale Pumpe (Impella, Abiomed Europe, Aachen) bringt man ebenfalls transfemoral ein und führt sie unter fluroskopischer Kontrolle retrograd über die Aortenklappe in den linken Ventrikel vor. Je nach Pumpengröße können 2,5 bis ca. 51/Min (je nach System) aus dem linken Ventrikel nicht pulsatil in die Aorta gepumpt werden. In zahlreichen Studien war das System sicher und hämodynamisch effektiv. Große randomisierte Studien zur Frage der Reduktion der Mortalität stehen noch aus. Zahlreiche kleinere Studien weisen darauf hin, dass der frühzeitige Einsatz des Systems vor allem bei Frauen die Mortalität im Vergleich zur vorhergesagten Mortalität reduzieren kann [55].

Beim Tandem-Heart-System (Cardiac Assist, Inc., Pittsburgh, PA, USA) platziert man über eine transseptale Punktion eine venöse Kanüle („outflow“) im linken Vorhof und eine Kanüle („backflow“) in die A. femoralis. Das System saugt oxygeniertes Blut aus dem linken Vorhof ab und führt es retrograd über die A. femoralis zurück. Die wesentlichen hämodynamischen Effekte sind so die Reduktion der linksventrikulären Vorlast und der Wandspannung. Das System kann mit einer Kapazität von 4,0l/min arbeiten. 


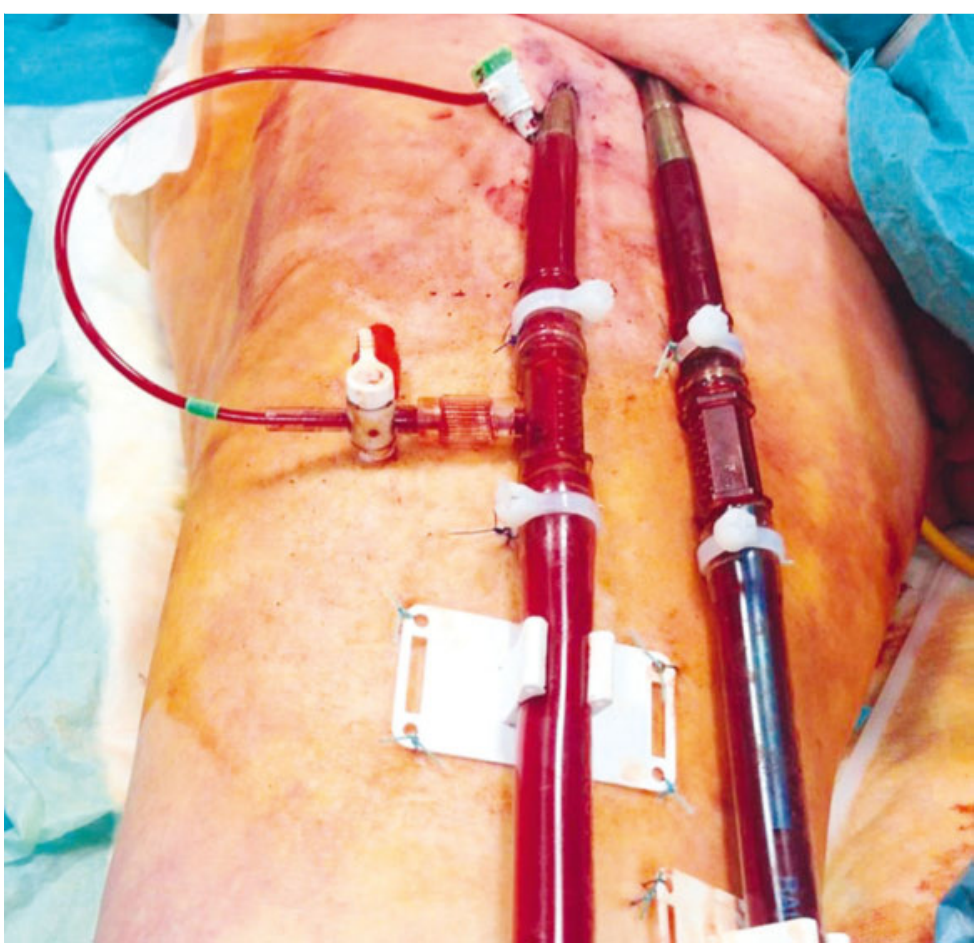

Abb. 3 Femorofemorale Kanülierung der linken Leiste bei ECMO mit Schleuse zur antegraden Perfusion des Beins.

Der auf konventionelle Maßnahmen refraktäre kardiogene Schock resultiert in einer anhaltenden Organminderperfusion. Hier besteht mit der venoarteriellen extrakorporalen Membranoxygenierung (va ECMO) die Möglichkeit, die Organperfusion zu erhalten und dabei den myokardialen Sauerstoffbedarf zu reduzieren.

Damit soll die Zeit bis zur Erholung des Myokards

(,bridge to recovery“) oder die Zeit bis zu einer inter- disziplinären Heart-Team-Entscheidung („,bridge to decision“) überbrückt werden [56]. Es stehen unterschiedliche Systeme unterschiedlicher Hersteller zur Verfügung. In aller Regel punktiert man mit speziellen dicklumigen Kanülen femorofemoral. Eine leistungsfähige Pumpe saugt dann venöses Blut im rechten Vorhof an, dekarboxyliert und oxygeniert es in einem Membranoxygenator und perfundiert es retrograd in die Aorta. In aller Regel ist aufgrund der Kanülendicke eine zusätzliche antegrade arterielle Perfusion des jeweiligen Beins erforderlich (Abb.3). Die Therapie ist ausgesprochen komplex und Bedarf einer hohen pflegerischen und ärztlichen Expertise. Sinnvollerweise wird sie in einem regionalen Konzept in Zusammenarbeit mit herzchirurgischen Abteilungen umgesetzt. Unter diesen Bedingungen sind Überlebensraten von Patienten im konventionell therapierefraktären kardiogenen Schock bis ca. 50\% erreichbar [57]. Wie auch für andere mechanische Unterstützungssysteme scheint zu gelten, dass der frühzeitige Einsatz vor einer fortgeschrittenen Mehrorgandysfunktion prognostisch relevant ist. Dem trägt das Konzept der extrakorporalen kardiopulmonalen Reanimation (eCPR) mittels ECMO Rechnung. Ein hoher Prozentsatz dieser Patienten erreichen wieder einen spontanen Kreislauf und können letztlich auch von der ECMO entwöhnt werden [58].

Eine aktuelle Übersicht über die verfügbaren mechanischen Unterstützungssysteme, ihre technischen Prinzipien und Funktionsweisen wurde kürzlich publiziert Abb. 4 [59].

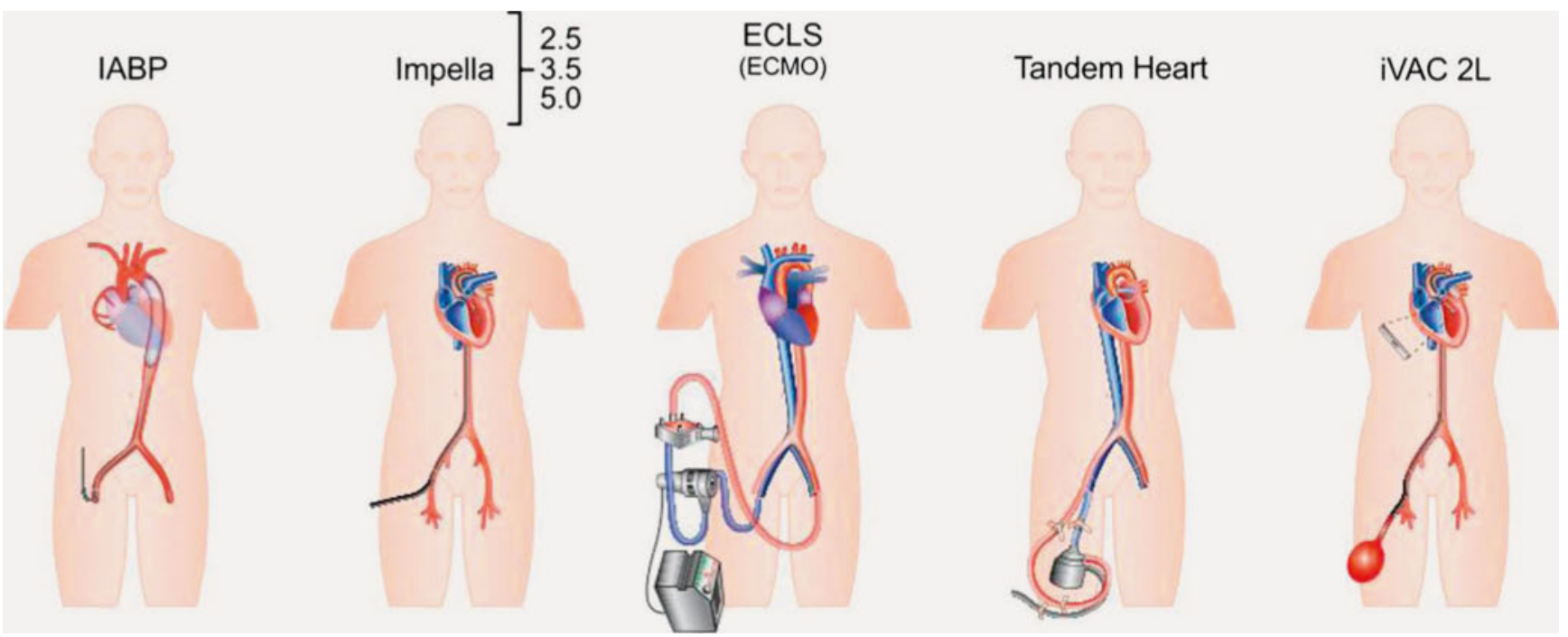

Abb. 4 Übersicht über die mechanischen Unterstützungssysteme (mit freundlicher Genehmigung von Eurolntervention [59]). 


\section{Kernaussagen}

- Die Prognose von Patienten mit ST-Strecken-Hebungsinfarkt hat sich in den vergangenen Jahren kontinuierlich verbessert. Entscheidend dafür sind die frühzeitige Diagnose, die Organisation der prä- und intrahospitalen Abläufe sowie die frühzeitige, komplette und anhaltende Reperfusion des Infarktgefäßes.

- Die prozeduralen Abläufe und Strategien sowie die periinterventionelle Begleit- therapie sind in großen kontrollierten Studien validiert und in Leitlinien eingearbeitet oder finden aktuell ihren Eingang in die Leitlinien.

- Eine große medizinische Herausforderung bleiben die Patienten im kardiogenen Schock trotz erfolgreicher Reperfusion. Die genauen pathophysiologischen Abläufe sind im Detail nicht klar.

- Es zeichnet sich zunehmend ab, dass mechanische Unterstützungssysteme einen Überlebensvorteil bieten können. Deren Einsatz ist teilweise komplex und benötigt eine hohe Expertise.

- Aus unserer Sicht sollten sich lokal in Krankenhäusern Schock-Teams etablieren, die sich regional vernetzen, um die teils aufwendigen Behandlungsverfahren optimal einsetzen zu können.

\section{Abstract}

Treatment and outcome of ST-segment elevation infarction (STEMI) has been advancing with the development of pharmacologic agents and mechanical revascularization that have reduced mortality. In patients presenting STEMI, primary percutaneous coronary intervention (PPCl) is recommended reperfusion therapy. Access site is an important procedural aspect related to the successful of a PPCI. However, the clinical outcome of patients with STEMI complicated by cardiogenic shock (CS) remains unsatisfactory despite high rates of early revascularization. Conservative management with inotropes belongs furthermore to the initial therapie, but nowadays mechanical circulatory support devices become widely used for intensive treatment of patients with circulatory collapse refractory to conventional treatment. The percutaneous assist systems most commonly used in cardiogenic shock complicating an ST-elevation myocardial infarction are the intra-aortic ballon pump (IABP), the Impella pump, v-a ECMO and the Tandem Heart.

Interessenkonflikt: Die Autoren erklären, dass kein Interessenkonflikt besteht.

\section{Über die Autoren}

\section{Harald Lapp}

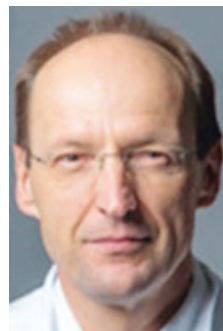

Prof. Dr. med. habil. 1979-1985 Studium der Humanmedizin der PhilipsUniversität Marburg. 1985 Approbation. 1986 Promotion. 1997 Facharzt für Innere Medizin. 1998 Facharzt für Kardiologie. 2004 Habilitation. 2005 Zusatzweiterbildung „Intensivmedizin“. Seit 2005 Chefarzt der Klinik für
Kardiologie/Internistische Intensivmedizin HELIOS Klinikum Erfurt GmbH. 2009 apl. Professur Universität Witten-Herdecke.

\section{Philipp Lauten}

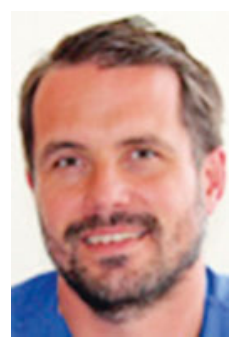

Dr. med. 1998-2004 Studium der Humanmedizin an der FriedrichSchiller-Universität Jena und Promotion. 2012 Facharzt für Innere Medizin sowie Zusatzbezeichnung Intensivmedizin. 2016 Facharzt für Kardiologie mit Tätigkeitsspektrum Intensivmedizin/invasive Kardiologie am HELIOS Klinikum Erfurt GmbH.

\section{Korrespondenzadresse}

Prof. Dr. Harald Lapp

3. Medizinische Klinik

Kardiologie, Internistische Intensivmedizin

HELIOS-Klinikum Erfurt

Nordhäuser Straße 74

99089 Erfurt

E-Mail: harald.lapp@helios-kliniken.de

\section{Literatur}

1 Writing Group M. Mozaffarian D, Benjamin EJ et al. Heart Disease and Stroke Statistics-2016 UpdateN: A Report From the American Heart Association. Circulation 2016; 133: e38 - e60

2 American College of Emergency P. Society for Cardiovascular A. Interventions. et al. 2013 ACCF/AHA guideline for the management of ST-elevation myocardial infarction: a report of the American College of Cardiology Foundation/American Heart Association Task Force on Practice Guidelines. J Am Coll Cardiol 2013; 61: e78-e140 
3 Task Force on the management of STseamiotESoC. Steg PG, James SK et al. ESC Guidelines for the management of acute myocardial infarction in patients presenting with ST-segment elevation. Eur Heart J 2012; 33: 2569-2619

4 Sgarbossa EB, Pinski SL, Barbagelata A et al. Electrocardiographic diagnosis of evolving acute myocardial infarction in the presence of left bundle-branch block. GUSTO-1 (Global Utilization of Streptokinase and Tissue Plasminogen Activator for Occluded Coronary Arteries) Investigators. N Engl J Med 1996; 334: 481-487

5 Cai Q, Mehta N, Sgarbossa EB et al. The left bundle-branch block puzzle in the 2013 ST-elevation myocardial infarction guideline: from falsely declaring emergency to denying reperfusion in a high-risk population. Are the Sgarbossa Criteria ready for prime time? Am Heart J 2013; 166: 409-413

6 Terkelsen CJ, Sorensen JT, Maeng M et al. System delay and mortality among patients with STEMI treated with primary percutaneous coronary intervention. JAMA 2010; 304: 763 771

7 Ndrepepa G, Kastrati A, Mehilli J et al. Mechanical reperfusion and long-term mortality in patients with acute myocardial infarction presenting 12 to 48 hours from onset of symptoms. JAMA 2009; 301: 487-488

8 Hochman JS, Lamas GA, Buller CE et al. Coronary intervention for persistent occlusion after myocardial infarction. N Engl J Med 2006; 355: 2395-407

9 Keeley EC, Boura JA, Grines CL. Primary angioplasty versus intravenous thrombolytic therapy for acute myocardial infarction: a quantitative review of 23 randomised trials. Lancet 2003; 361: 13-20

10 Widimsky P, Budesinsky T, Vorac D et al. Long distance transport for primary angioplasty vs immediate thrombolysis in acute myocardial infarction. Final results of the randomized national multicentre trial - PRAGUE-2. Eur Heart J 2003; 24: $94-104$

11 Andersen HR, Nielsen TT, Rasmussen K et al. A comparison of coronary angioplasty with fibrinolytic therapy in acute myocardial infarction. N Engl J Med 2003; 349: 733 - 742

12 Kastrati A, Dibra A, Spaulding C et al. Meta-analysis of randomized trials on drug-eluting stents vs. bare-metal stents in patients with acute myocardial infarction. Eur Heart J 2007; 28: $2706-2713$

13 Alfonso F, Cuesta J, Bastante T et al. Bioresorbable vascular scaffolds in patients with acute myocardial infarction: a new step forward to optimized reperfusion? J Thorac Dis 2016; 8: E417-E423

14 Sabate M, Windecker S, Iniguez A et al. Everolimus-eluting bioresorbable stent vs. durable polymer everolimus-eluting metallic stent in patients with ST-segment elevation myocardial infarction: results of the randomized ABSORB ST-segment elevation myocardial infarction-TROFI II trial. Eur Heart J 2016; 37: $229-240$

15 Eikelboom JW, Mehta SR, Anand SS et al. Adverse impact of bleeding on prognosis in patients with acute coronary syndromes. Circulation 2006; 114: 774-782

16 Baklanov DV, Kaltenbach LA, Marso SP et al. The prevalence and outcomes of transradial percutaneous coronary intervention for ST-segment elevation myocardial infarction: analysis from the National Cardiovascular Data Registry (2007 to 2011). J Am Coll Cardiol 2013; 61: 420-426
17 Romagnoli E, Biondi-Zoccai G, Sciahbasi A et al. Radial versus femoral randomized investigation in ST-segment elevation acute coronary syndrome: the RIFLE-STEACS (Radial Versus Femoral Randomized Investigation in ST-Elevation Acute Coronary Syndrome) study. J Am Coll Cardiol 2012; 60: 2481 2489

18 Johnman C, Pell JP, Mackay DF et al. Clinical outcomes following radial versus femoral artery access in primary or rescue percutaneous coronary intervention in Scotland: retrospective cohort study of 4534 patients. Heart 2012; 98: $552-557$

19 Svilaas T, Vlaar PJ, van der Horst IC et al. Thrombus aspiration during primary percutaneous coronary intervention. $\mathrm{N}$ Engl J Med 2008; 358: 557-567

20 Vlaar PJ, Svilaas T, van der Horst IC et al. Cardiac death and reinfarction after 1 year in the Thrombus Aspiration during Percutaneous coronary intervention in Acute myocardial infarction Study (TAPAS): a 1-year follow-up study. Lancet 2008; 371: 1915-1920

21 Frobert O, Lagerqvist B, Olivecrona GK et al. Thrombus aspiration during ST-segment elevation myocardial infarction. N Engl J Med 2013; 369: 1587 - 1597

22 Lagerqvist B, Frobert O, Olivecrona GK et al. Outcomes 1 year after thrombus aspiration for myocardial infarction. $N$ Engl J Med 2014; 371: $1111-1120$

23 Stone GW, Maehara A, Witzenbichler B et al. Intracoronary abciximab and aspiration thrombectomy in patients with large anterior myocardial infarction: the INFUSE-AMI randomized trial. JAMA 2012; 307: 1817-1826

24 Elgendy IY, Huo T, Bhatt DL et al. Is Aspiration Thrombectomy Beneficial in Patients Undergoing Primary Percutaneous Coronary Intervention? Meta-Analysis of Randomized Trials Circ Cardiovasc Interv 2015; 8: e002258

25 Park DW, Clare RM, Schulte PJ et al. Extent, location, and clinical significance of non-infarct-related coronary artery disease among patients with ST-elevation myocardial infarction. JAMA 2014; 312: 2019-2027

26 Hannan EL, Samadashvili Z, Walford G et al. Culprit vessel percutaneous coronary intervention versus multivessel and staged percutaneous coronary intervention for ST-segment elevation myocardial infarction patients with multivessel disease. JACC Cardiovasc Interv 2010; 3: 22-31

27 Vlaar PJ, Mahmoud KD, Holmes DRJr et al. Culprit vessel only versus multivessel and staged percutaneous coronary intervention for multivessel disease in patients presenting with STsegment elevation myocardial infarction: a pairwise and network meta-analysis. J Am Coll Cardiol 2011; 58: 692-703

28 Engstrom T, Kelbaek H, Helqvist $\mathrm{S}$ et al. Complete revascularisation versus treatment of the culprit lesion only in patients with ST-segment elevation myocardial infarction and multivessel disease (DANAMI-3-PRIMULTI): an open-label, randomised controlled trial. Lancet 2015; 386: 665-671

29 Gershlick AH, Khan JN, Kelly DJ et al. Randomized trial of complete versus lesion-only revascularization in patients undergoing primary percutaneous coronary intervention for STEMI and multivessel disease: the CvLPRIT trial. J Am Coll Cardiol 2015; 65: $963-972$

30 Manari A, Varani E, Guastaroba P et al. Long-term outcome in patients with ST segment elevation myocardial infarction and multivessel disease treated with culprit-only, immediate, or staged multivessel percutaneous revascularization strategies: 
Insights from the REAL registry. Catheter Cardiovasc Interv 2014; 84: $912-922$

31 Wald DS, Morris JK, Wald NJ et al. Randomized trial of preventive angioplasty in myocardial infarction. N Engl J Med 2013; 369: $1115-1123$

32 Thiele H, Desch S, Piek JJ et al. Multivessel versus culprit lesion only percutaneous revascularization plus potential staged revascularization in patients with acute myocardial infarction complicated by cardiogenic shock: Design and rationale of CULPRIT-SHOCK trial. Am Heart J 2016; 172: $160-169$

33 Wiviott SD, Braunwald E, McCabe $\mathrm{CH}$ et al. Prasugrel versus clopidogrel in patients with acute coronary syndromes. N Engl J Med 2007; 357: 2001 - 2015

34 Wallentin L, Becker RC, Budaj A et al. Ticagrelor versus clopidogrel in patients with acute coronary syndromes. N Engl J Med 2009; 361: 1045-1057

35 De Backer O, Ratcovich H, Biasco L et al. Prehospital administration of P2Y12 inhibitors and early coronary reperfusion in primary PCI: an observational comparative study. Thromb Haemost 2015; 114: 623-631

36 Montalescot G, van 't Hof AW, Lapostolle F et al. Prehospital ticagrelor in ST-segment elevation myocardial infarction. N Engl J Med 2014; 371: 1016 - 1027

37 Bhatt DL, Stone GW, Mahaffey KW et al. Effect of platelet inhibition with cangrelor during $\mathrm{PCl}$ on ischemic events. N Engl J Med 2013; 368: $1303-1313$

38 Bonaca MP, Bhatt DL, Cohen M et al. Long-term use of ticagrelor in patients with prior myocardial infarction. $N$ Engl J Med 2015; 372: 1791 - 800

39 Bonaca MP, Bhatt DL, Steg PG et al. Ischaemic risk and efficacy of ticagrelor in relation to time from $\mathrm{P} 2 \mathrm{Y} 12$ inhibitor withdrawal in patients with prior myocardial infarction: insights from PEGASUS-TIMI 54. Eur Heart J 2016; 37: 1133 - 1142

40 Udell JA, Bonaca MP, Collet JP et al. Long-term dual antiplatelet therapy for secondary prevention of cardiovascular events in the subgroup of patients with previous myocardial infarction: a collaborative meta-analysis of randomized trials. Eur Heart ] 2016; 37: 390 - 399

41 Yeh RW, Kereiakes DJ, Steg PG et al. Benefits and Risks of Extended Duration Dual Antiplatelet Therapy After PCl in Patients With and Without Acute Myocardial Infarction. J Am Coll Cardiol 2015; 65: 2211-2221

42 Yeh RW, Secemsky EA, Kereiakes DJ et al. Development and Validation of a Prediction Rule for Benefit and Harm of Dual Antiplatelet Therapy Beyond 1 Year After Percutaneous Coronary Intervention. JAMA 2016; 315: 1735 - 1749

43 Montalescot G, Zeymer U, Silvain J et al. Intravenous enoxaparin or unfractionated heparin in primary percutaneous coronary intervention for ST-elevation myocardial infarction: the international randomised open-label ATOLL trial. Lancet 2011; 378: $693-703$

44 Mehran R, Lansky AJ, Witzenbichler B et al. Bivalirudin in patients undergoing primary angioplasty for acute myocardial infarction (HORIZONS-AMI): 1-year results of a randomised controlled trial. Lancet 2009; 374: 1149-1159

45 Ducrocq G, Steg PG, Van't Hof A et al. Utility of post-procedural anticoagulation after primary PCI for STEMI: insights from a pooled analysis of the HORIZONS-AMI and EUROMAX trials. Eur Heart J Acute Cardiovasc Care 2016: Epup ahead of print
46 Kern KB, Rahman O. Emergent percutaneous coronary intervention for resuscitated victims of out-of-hospital cardiac arrest. Catheter Cardiovasc Interv 2010; 75: 616-624

47 Katz JN, Stebbins AL, Alexander JH et al. Predictors of 30-day mortality in patients with refractory cardiogenic shock following acute myocardial infarction despite a patent infarct artery. Am Heart J 2009; 158: 680-687

48 Hochman JS, Sleeper LA, Webb JG et al. Early revascularization in acute myocardial infarction complicated by cardiogenic shock. SHOCK Investigators. Should We Emergently Revascularize Occluded Coronaries for Cardiogenic Shock. N Engl J Med 1999; 341: 625-634

49 Dzavik V, Sleeper LA, Cocke TP et al. Early revascularization is associated with improved survival in elderly patients with acute myocardial infarction complicated by cardiogenic shock: a report from the SHOCK Trial Registry. Eur Heart J 2003; 24: $828-837$

50 Tarvasmaki T, Lassus J, Varpula M et al. Current real-life use of vasopressors and inotropes in cardiogenic shock - adrenaline use is associated with excess organ injury and mortality. Crit Care 2016; 20: 208

51 Arbel Y, Mass R, Ziv-Baran T et al. Prognostic implications of fluid balance in ST elevation myocardial infarction complicated by cardiogenic shock. Eur Heart J Acute Cardiovasc Care 2016: Epup ahead of print

52 Harjola VP, Mebazaa A, Celutkiene J et al. Contemporary management of acute right ventricular failure: a statement from the Heart Failure Association and the Working Group on Pulmonary Circulation and Right Ventricular Function of the European Society of Cardiology. Eur J Heart Fail 2016; 18: $226-241$

53 Acharya D, Loyaga-Rendon RY, Pamboukian SV et al. Ventricular Assist Device in Acute Myocardial Infarction. J Am Coll Cardiol 2016; 67: $1871-1880$

54 Thiele H, Zeymer U, Neumann FJ et al. Intra-aortic balloon counterpulsation in acute myocardial infarction complicated by cardiogenic shock (IABP-SHOCK II): final 12 month results of a randomised, open-label trial. Lancet 2013; 382: 1638 1645

55 Joseph SM, Brisco MA, Colvin M et al. Women With Cardiogenic Shock Derive Greater Benefit From Early Mechanical Circulatory Support: An Update From the cVAD Registry. J Interv Cardiol 2016; 29: $248-256$

56 Burrell AJ, Pellegrino VA, Wolfe R et al. Long-term survival of adults with cardiogenic shock after venoarterial extracorporeal membrane oxygenation. J Crit Care 2015; 30: 949-956

57 Dini CS, Lazzeri C, Chiostri M et al. A local network for extracorporeal membrane oxygenation in refractory cardiogenic shock. Acute Card Care 2016: Epup ahead of print

58 Spangenberg T, Meincke F, Brooks S et al. "Shock and Go?" extracorporeal cardio-pulmonary resuscitation in the goldenhour of ROSC Catheter Cardiovasc Interv 2016: Epup ahead of print

59 Blumenstein J, de Waha S, Thiele H. Percutaneous ventricular assist devices and extracorporeal life support: current applications. Eurolntervention 2016; 12: X61 - X70 


\section{CME-Fragen}

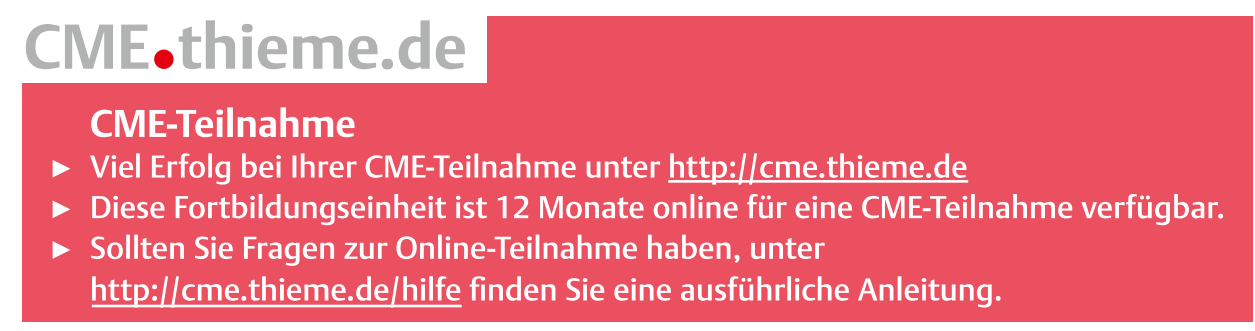

1 Welche der folgenden Aussagen zur Diagnostik des STEMI ist richtig?

A Bei typischen EKG-Veränderungen, die die STEMI-Kriterien erfüllen, sind zur weiteren Therapie pathologisch veränderte Biomarker notwendig.

B Die Diagnose eines STEMI ist bei Linksschenkelblock nicht möglich.

C Für die Diagnose eines STEMI sind ST-Streckenveränderungen im 12-Kanal-EKG wesentlich.

D Die Diagnose eines akuten Myokardinfarkt beruht u. a. auf neuen ST-Strecken-Hebungen $\geq 0,1 \mathrm{mV}$ am J-Punkt in allen Ableitungen außer $\mathrm{V}_{2}$ und $\mathrm{V}_{3}$.

E Hochsensitives Troponin (T oder I) ist vor Reperfusion als Biomarker der Wahl für eine Myokardnekrose auch bei klarer Anamnese und eindeutigem EKG immer erforderlich

2 Welche der folgenden Aussagen zur Therapie eines STEMI ist nicht richtig?

A Die symptomatische Behandlung von Schmerz, Brechreiz, Angst sowie Luftnot sind wichtige Maßnahmen bereits in der prähospitalen Phase.

B Patienten mit einem Myokardinfarkt sollten immer Sauerstoff via Sonde oder Maske erhalten.

C Vom ersten medizinischen Kontakt bis zur Ballondilatation dürfen nicht mehr als 90 Minuten vergehen.

D Die „door-to-balloon“-Zeit darf nicht mehr als 60 Minuten betragen.

E Patienten mit der eindeutigen Diagnose eines STEMI sollten direkt vom Rettungsdienst ins Katheterlabor verbracht werden.

3 Welche der folgenden Aussagen zum infarktbedingten kardiogenen Schock ist richtig?
A Ein kardiogener Schock entsteht bei ca. $20 \%$ aller Patienten mit STEMI.
B Die Mortalität liegt bei etwa $80 \%$.
C Patienten über 75 Jahre sind besonders häufig betroffen.
D Die interventionelle Revaskularisation ist der Therapie mit Thrombolyse und IABP unterlegen.
E Die Schocksymptome manifestieren sich vor den eigentlichen Infarktzeichen.

4 Welche der folgenden Aussagen ist richtig?
A Der Einsatz von DES der 2. Generation führt zu einer erhöhten Rate an Stentthrombosen.
B Beim STEMI ist nach Implantation eines Koronarstents eine duale Plättchenhemmung für 6 Monate ausreichend.
C Prasugrel ist bei Patienten nach Schlaganfall oder TIA in der Anamnese kontraindiziert.
D Die prähospitale Gabe von Prasugrel bzw. Ticagrelor bedeutet einen deutlichen klinischen Gewinn für die Patienten.
E Bei verlängerter Gabe von Ticagrelor ist die Rate an Blutungen nicht erhöht.

5 Welche der folgenden Aussagen zu Thrombozytenaggregationshemmern ist nicht richtig?

A Die Dosierung von Azetylsalizylsäure (ASS) beträgt bei der dualen Plättchenhemmung $100 \mathrm{mg}$.

B Clopidogrel, Prasugrel sowie Ticagrelor sind ADP-(P2Y 12$)$-Rezeptorantagonisten.

C Die Halbwertszeit des P2Y12-Rezeptorhemmers Cangrelor beträgt etwa 5 Minuten.

D Die Hemmung der Thrombozytenaggregation ist nur in der Initialbehandlung des ACS ein bedeutender Bestandteil der medikamentösen Therapie.

E Die orale Startdosis von Ticagrelor im Rahmen der dualen Plättchenhemmung liegt bei $180 \mathrm{mg}$. 


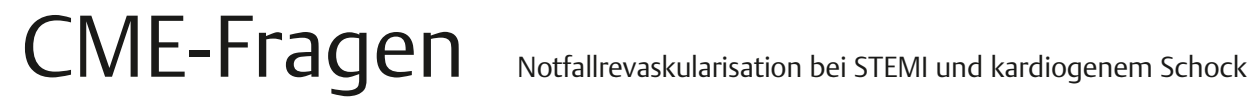

6 Welche der folgenden Faktoren ist bei der Nutzen-Risiko-Abwägung einer verlängerten DAPT kein Risikofaktor eines ischämischen Ereignisses?
A fortgeschrittenes Alter
B ausgedehnte $\mathrm{KHL}$
C Niereninsuffizienz
D weibliches Geschlecht
E eingeschränkte linksventrikuläre Funktion (LVEF $<40 \%)$

7 Welche der folgenden Aussagen zum infarktbedingten kardiogenen Schock ist richtig?

A Die Indikation zu mechanischen Unterstützungsmaßnahmen wird rein klinisch gestellt.

B Mit Noradrenalin wird ein arterieller Mitteldruck $>60 \mathrm{mmHg}$ angestrebt.

C Eine IABP gehört fest zum Therapieregime.

D Eine akute Mitralklappeninsuffizienz bei Papillarmuskelruptur, der akute Ventrikelseptumdefekt oder die Ruptur der freien Wand treten typischerweise in den ersten 24 Stunden des STEMI auf.

E Eine Volumentherapie verbietet sich bei einem kardialen Low-Output-Syndrom.

8 Welche der folgenden Aussagen zur Therapie des kardiogenen Schocks ist nicht richtig?

A Dobutamin und Phosphodiesterasehemmer haben eine positiv inotrope Wirkung.

B Der Vasopressor der Wahl ist Noradrenalin.

C Die Flüssigkeitsbilanz sollte beim kardiogenen Schock immer positiv sein.

D Auf eine initiale Bolusgabe sollte bei Levosimendan verzichtet werden.

E Die Narkoseeinleitung erfordert viel Erfahrung, um zusätzliche akute Hypotonien zu vermeiden.

9 Welche der folgenden Aussagen ist richtig?

A Eine maschinelle Beatmung sollte im kardiogenen Schock unbedingt vermieden werden.

B Die Prognose von Patienten mit ST-Strecken-Hebungsinfarkt hat sich in den vergangenen Jahren kontinuierlich verbessert.

C Die nicht invasive Beatmung sollte der Intubation und Beatmung vorgezogen werden.

D Passagere mechanische Unterstützungssysteme haben beim rechtsventrikulär bedingten kardiogenen Schock keinen entscheidenden Einfluss auf den Verlauf.

E Für die Indikationsstellung pharmakologischer Maßnahmen sind die Ejektionsfraktion und die Füllungsdrucke irrelevant.

10 Welche der folgenden Aussagen zu Assist-Systemen ist richtig?

A Zur Behandlung des kardiogenen Schocks existieren ausschließlich Systeme zur Unterstützung des linken Ventrikels.

B Die IABP führt zu einer systolischen Augmentation des Blutflusses der Aorta und erhöht damit das Herzzeitvolumen.

C Systeme wie das Tandem Heart reduzieren vor allem die Vorlast des rechten Ventrikels.

D Für die mikroaxiale Pumpe ist die Reduktion der Mortalität in zahlreichen Studien nachgewiesen.

E Über die ECMO kann deoxygeniertes Blut mit Sauerstoff gesättigt werden. 